\title{
Azathioprine Hypersensitivity Syndrome
}

JAMES FRANK, BS, Oregon Health and Science University, School of Medicine; NICOLE FETT, MD, MSCE, Department of Dermatology, Oregon Health and Science University, Portland, Oregon, USA. Address correspondence to Dr. N. Fett, Associate Professor of Dermatology, Oregon Health and Science University, 3303 SW Bond Ave., Portland, Oregon 97239, USA. E-mail: fett@ohsu.edu. Ethics approval is not required by the authors’ institution. Patient permission was obtained. J Rheumatol 2017;44:1876-7; doi:10.3899/jrheum.170066

Azathioprine hypersensitivity syndrome (AHS) is a rare yet life-threatening clinical phenomenon seen in about $2 \%$ of patients within weeks of initiating therapy. Clinical features include constitutional symptoms with or without a cutaneous reaction, and a high index of suspicion is required to avoid a delay in diagnosis ${ }^{1,2}$. Less commonly, liver and renal dysfunction, hypotension, and shock may occur ${ }^{1}$. Laboratory abnormalities include neutrophilia, leukocytosis, anemia, elevated inflammatory markers, and rarely, positive antineutrophilic cytoplasmic antibodies ${ }^{2,3}$.

A woman in her 20 s with systemic lupus erythematosus presented with a 3-week history of rash. The eruption started with scattered nontender, pruritic pustules on the right leg and then generalized to the torso and extremities (Figure 1). The patient reported concurrent fevers, fatigue, nausea, myalgias, and arthralgias. Laboratory evaluation revealed neutrophilia, mild anemia, stable proteinuria, and normal comprehensive metabolic panel. Six weeks prior to presentation, azathioprine (AZA) was started. Biopsy showed subcorneal collection of neutrophils with diffuse dermal neutrophilic infiltrate without evidence of vasculitis (Figure 2). A diagnosis of AHS was made. AZA was substituted with mycophenolate mofetil, resulting in complete resolution on Day 10 followup.

While the exact etiology of AHS remains unknown, current studies have suggested a type III or IV immune-mediated reaction ${ }^{1,2}$. Given the mortality associated with AHS, for patients who present with systemic and cutaneous symptoms while taking AZA, the safest course is to stop taking it. Upon AZA cessation, complete resolution typically occurs within a week ${ }^{2,4}$. Rechallenge is not recommended because of an increased risk of morbidity and mortality ${ }^{4}$.

\section{REFERENCES}

1. Bidinger JJ, Sky K, Battafarano DF, Henning JS. The cutaneous and systemic manifestations of azathioprine hypersensitivity syndrome. J Am Acad Dermatol 2011;65:184-91.

2. Cyrus N, Stavert R, Mason AR, Ko CJ, Choi JN. Neutrophilic dermatosis after azathioprine exposure. JAMA Dermatol 2013;149:592-7

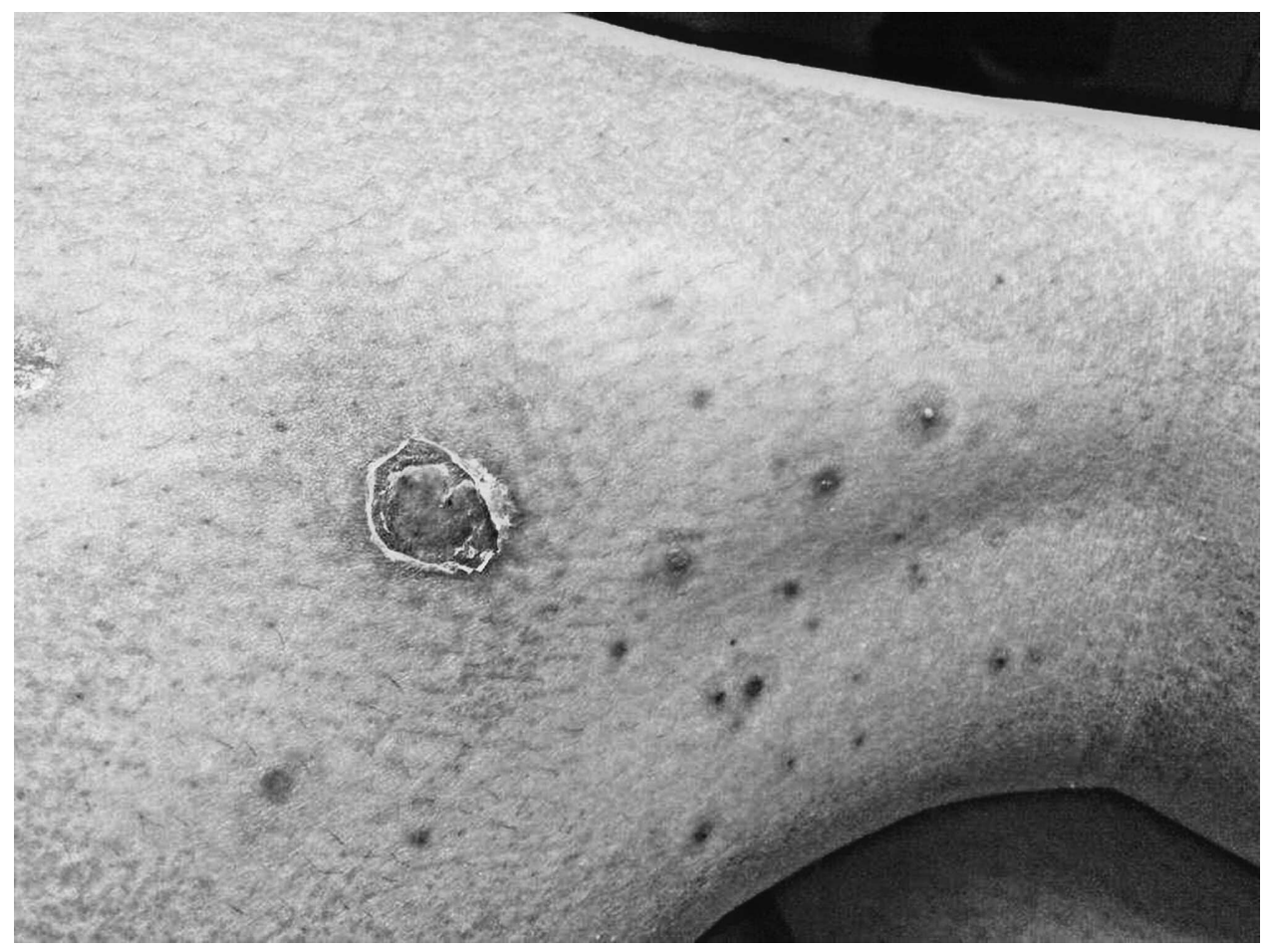

Figure 1. Multiple erythematous pustules and nodules on the right leg. 


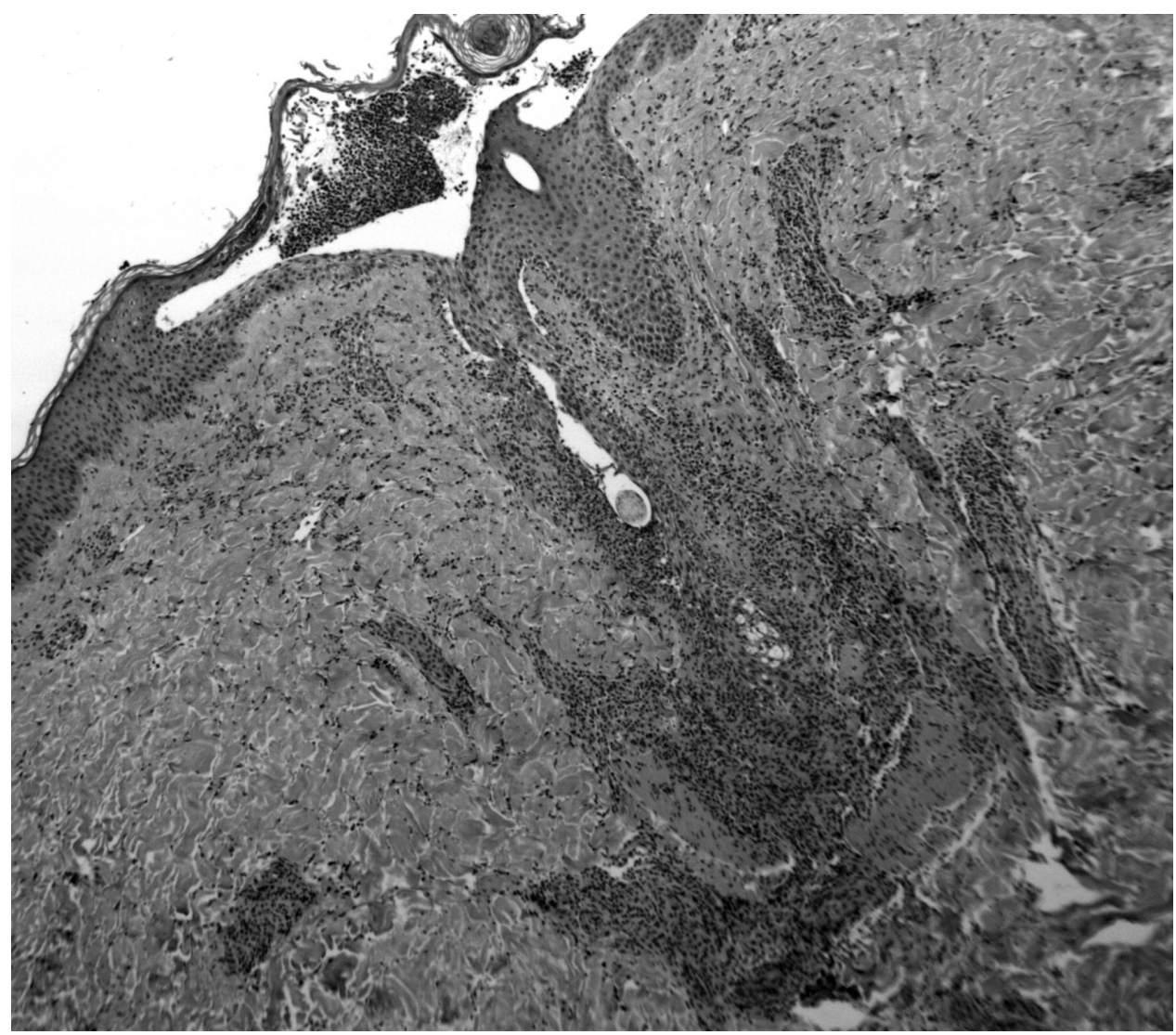

Figure 2. Histopathologic image showing a subcorneal collection of neutrophils with diffuse dermal neutrophilic infiltrate $(\mathrm{H} \& \mathrm{E}$, original magnification $\times 10)$.

3. Choonhakarn C, Chaowattanapanit S. Azathioprine-induced Sweet's syndrome and published work review. J Dermatol 2013;40:267-71.

4. McNally A, Ibbetson J, Sidhu S. Azathioprine-induced Sweet's syndrome: A case series and review of the literature. Australas J Dermatol 2017;58:53-7. 